\title{
Monte Carlo Simulation of HEMT based on Self- Consistent Method
}

\author{
H. UENO ${ }^{\mathrm{a}}$, S. YAMAKAWA ${ }^{\mathrm{a}}$, C. HAMAGUCHI ${ }^{\mathrm{a}}$ and K. MIYATSUJI ${ }^{\mathrm{b}}$ \\ ${ }^{a}$ Department of Electronic Engineering, Faculty of Engineering, Osaka University, Suita City, Osaka 565, Japan \\ ${ }^{\mathrm{b}}$ Electronics Research Laboratory, Matsushita Electronics Corporation, Takatsuki City, Osaka 569, Japan
}

\begin{abstract}
Device simulation of HEMT (High Electron Mobility Transistor) has been carried out by ensemble Monte Carlo simulation, where two-dimensional motion of electrons confined in $\mathrm{GaAs}$ region at $\mathrm{GaAs} / \mathrm{AlGaAs}$ heterointerface is taken into account. In order to develop a realistic device simulator of HEMT, we divide the channel region in meshes, and Schrödinger and Poisson equations are solved self-consistently to obtain electronic states of the twodimensional electrons in a mesh. In addition the real space transfer from GaAs layer into $\mathrm{AlGaAs}$ barrier layer is taken into account.
\end{abstract}

\section{INTRODUCTION}

Device simulations of HEMT reported so far[1],[2] assume simplified models rather than reported here. Tomizawa et al.[1] divided the device into twodimensional electron region and three-dimensional electron region by using a simplified relation without taking into account the energy of electrons at high fields. Ravaioli and Ferry[2] took into account two subband only. Zhou and Ferry[3] have recently reported numerical simulation of ultra-submicrometer HEMTs based upon a set of quantum moment equations.

Several authors reported the electron transport properties in $\mathrm{GaAs} / \mathrm{AlGaAs}$ single heterostructures using Monte Carlo simulation[1],[2],[4]. The analysis is based on the electronphonon interaction model proposed by Price[5], where the quantized electrons (2DEG) are assumed to be scattered by bulk phonons and thus the wave functions calculated by the selfconsistent analysis are used to evaluated the scattering rate. In our previous simulation of HEMT the electric field parallel to channel is treated as uniform. In real device, however, the sheet electron density in the channel is not homogeneous and the electric field along the channel is not uniform, higher near the drain. In addition at high electric fields electrons are heated to form hot electrons which behave as threedimensional electrons rather than 2DEG. Another important effect is the real space transfer of hot electrons between the GaAs layer and the AlGaAs barrier. Here we present Monte Carlo device simulator taking into account of non-uniform channel and real space transfer.

\section{THEORETICAL MODELS}

\section{Calculation of Scattering Rate}

We employ the self-consistent calculation method for estimating the subband structures of GaAs layer. In the energy band structure of $\mathrm{GaAs}$, the lower conduction band minima are at $\Gamma, L$ and $X$ valleys. We calcu- 
lated the eigen states of electrons in $\mathrm{GaAs} / \mathrm{AlGaAs}$ hetero-junction assuming that the electrons in the three valleys are two-dimensional. In the case of GaAs/AlGaAs hetero-junction, we have to note that calculations should be carried out including electron transport in the spacer layer of $\mathrm{AlGaAs}$ because the barrier height between GaAs and AlGaAs is not so high to prevent the real space transfer. The electronic states of 2DEG in the $r$ valley are calculated by solving the Schrödinger equation and Poisson equation self-consistently [6].

$$
\begin{gathered}
{\left[-\frac{\hbar^{2}}{2 m^{*}} \frac{\mathrm{d}^{2}}{\mathrm{~d} z^{2}}-e \phi(z)\right] \zeta_{i}(z)=E_{i} \zeta_{i}(z),} \\
\frac{\mathrm{d}^{2} \phi}{\mathrm{d} z^{2}}=-\frac{1}{\kappa \varepsilon_{0}}\left[\rho_{\mathrm{depl}}(z)-e \sum_{i} N_{i}\left|\zeta_{i}(z)\right|^{2}\right],
\end{gathered}
$$

where $m^{*}$ is the effective mass in $\Gamma$ valley. $\phi(z)$ is the electrostatic potential, $\rho_{\text {depl }}$ the charge density of the depletion layer, $\kappa \varepsilon_{0}$ the static dielectric constant of GaAs, $N_{s}$ the electron sheet density. Since the electrons in the interface of hetero-junction form twodimensional states, Monte Carlo simulation requires the use of the scattering rate of the 2DEG interacting with three-dimensional phonons formulated by Price[5]. In our simulation, we take into account acoustic phonon, polar optical phonon, ionized impurity and inter valley phonon scatterings.

\section{Device Simulation}

In the previous $2 \mathrm{DEG}$ simulator the channel region is treated as uniform between the source and drain. In the real device, however, the channel region is not uniform. Another important factor for HEMT device simulation is to take into account the real space transfer from GaAs layer into AlGaAs barrier layer. In addition to these, electrons with high energy beyond the barrier height are not confined in the confinement potential but behave as three-dimensional electrons.

Figure 1 shows the device model used for the Monte Carlo simulation, where the channel length is $0.5 \mu \mathrm{m}$ and the thickness of spacer layer of $\mathrm{AlGaAs}$ is $100 \AA$. In order to simulate electron transport in nonuniform channel, we employed the following two

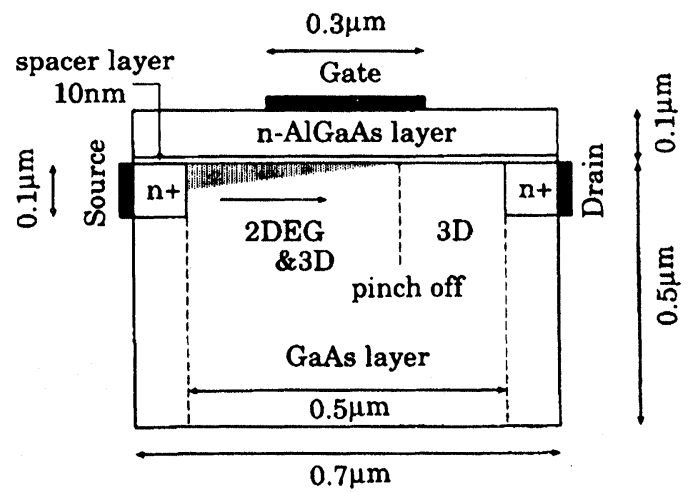

FIGURE 1 HEMT device structure used for the Monte Carlo simulation

steps. First, we evaluate the initial condition. We estimate the sheet electron density at each position between the source and drain using the current continuity relation along the channel. The drain current is estimated as

$$
\begin{gathered}
I_{D}=\frac{W}{L} \mu C\left[f\left(\phi_{s L}\right)-f\left(\phi_{s 0}\right)\right] \\
f\left(\phi_{s}\right)=\left(V_{G}+\frac{1}{\beta}\right) \phi_{s}-\frac{1}{2} \phi_{s}^{2}-\frac{2}{3} a \phi_{s}^{3 / 2} \\
+\frac{1}{\beta} a \sqrt{\phi_{s}} \quad\left(a=\frac{\sqrt{2 \kappa \varepsilon_{0} q N_{A}}}{C}\right),
\end{gathered}
$$

where $\mathrm{W}$ is the channel width, $\mathrm{L}$ the channel length, $\mathrm{C}$ the capacitance of AlGaAs layer and $\beta=q / k_{B} T=\mu / D$ (Einstein relation). $\phi_{s 0}$ and $\phi_{s L}$ are surface potentials at the edge of source and drain. At the point of $x$ measured from the source edge, the current is given by

$$
I_{D}=\frac{W}{x} \mu C\left[f\left(\phi_{s}(x)\right)-f\left(\phi_{s 0}\right)\right],
$$

where $\phi_{s}(x)$ is the surface potential at the point of $x$. Since the current is uniform along the channel, we can estimate $\phi_{s}(x)$ and $N_{s}(x)$.

Second, Monte Carlo simulation was carried out by evaluating the scattering rates of the electronic states in each mesh. Then two-dimensional Poisson equation was solved to obtain the potential distribution in the device. The Monte Carlo procedures were repeated until we obtain a steady state at an applied gate and drain voltages. We assume electrons are twodimensional in the channel region from the source to 
the pinch off point and three-dimensional beyond the pinch off point at the beginning of the simulation. At high electric fields, however, the two-dimensional electrons are heated to form hot electrons which are not confined in the region near the interface but behave as three-dimensional electrons. Therefore, an electron state in the channel region is defined as

$$
\left\{\begin{array}{l}
E_{\|}+E_{i}<E_{\mathrm{th}} \rightarrow 2 \mathrm{D} \\
E_{\|}+E_{i}>E_{\mathrm{th}} \rightarrow 3 \mathrm{D}
\end{array}\right.
$$

where $E_{\mathrm{i}}$ is the subband level and $E_{\mathrm{th}}$ the threshold energy. In the present simulation $E_{\mathrm{th}}$ is taken to be the conduction band discontinuity between GaAs layer and $\mathrm{AlGaAs}$ barrier layer at each valley. When the electronic state changes from two-dimensional to three-dimensional, we assume that the subband energy transfer to the kinetic energy in the $z$ direction.

$$
\frac{\hbar^{2}}{2 m^{*}} k_{z}^{2}=E_{n}
$$

Also real space transfer from $\mathrm{GaAs}$ layer into $\mathrm{AlGaAs}$ barrier layer is treated in a similar fashion. When an electron changes from three-dimensional to twodimensional, the electron is assumed to be confined in a subband $E_{\mathrm{i}}$ given by

$$
E_{i}<\varepsilon_{z}<E_{i+1} \text {. }
$$

\section{CALCULATION AND DISCUSSION}

In the present simulation we take 20,000 electrons in the source and drain region, and the simulation carried out in the time scale up to 30 ps by $1 \mathrm{fs}$ step.

Figures 2 and 3 show the electron distribution obtained by the present simulation, where the gate voltages are $0.5 \mathrm{~V}$ and $1.0 \mathrm{~V}$, respectively, and drain voltage is $0.3 \mathrm{~V}$. When the gate voltage is $0.5 \mathrm{~V}$, there are few electrons transferred from GaAs layer into $\mathrm{AlGaAs}$ barrier layer. However, more electrons are transferred from $\mathrm{GaAs}$ layer to $\mathrm{AlGaAs}$ barrier layer when gate voltage is increased to $1.0 \mathrm{~V}$. Figure 4 shows the electron drift velocity averaged in the meshes along $z$ direction as a function of the position along the channel. When drain voltage is low, the drift velocity between the source and the drain increases in

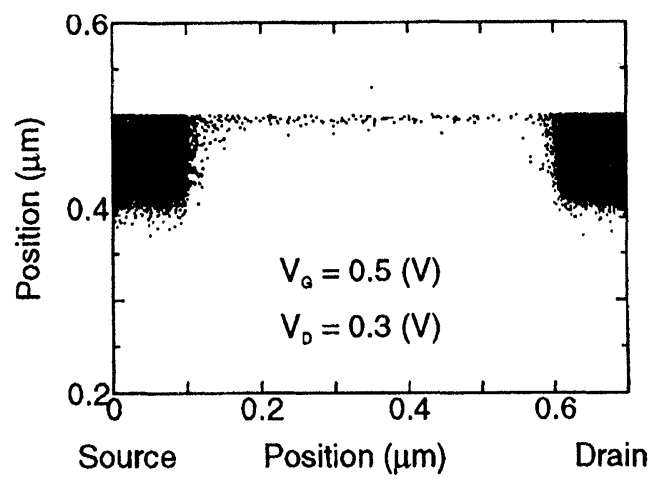

FIGURE 2 Electron distribution obtained by the Monte Carlo simulation in the HEMT for gate voltage $0.5 \mathrm{~V}$ and drain voltage $0.3 \mathrm{~V}$

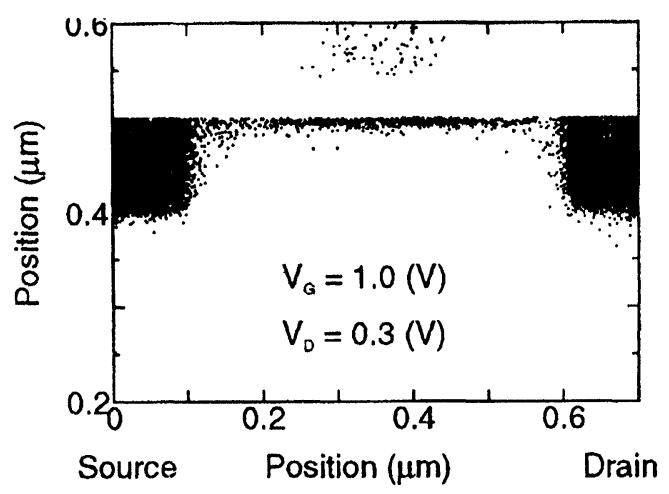

FIGURE 3 Electron distribution obtained by the Monte Carlo simulation in the HEMT for gate voltage $1.0 \mathrm{~V}$ and drain voltage $0.3 \mathrm{~V}$

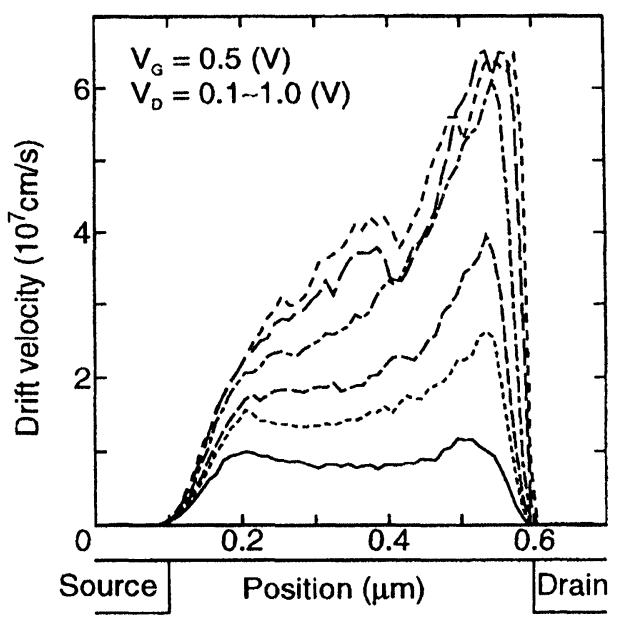

FIGURE 4 Average electron drift velocity in the channel region is plotted as a function of position for gate voltage $0.5 \mathrm{~V}$ 
proportion to the field parallel to the surface. As the drain voltage becomes high, the velocity saturation is observed near the drain region.

Figure 5 shows $I_{D}$ versus $V_{D}$ characteristics estimated from the present simulation. Although experimental results are not available for comparison, the present results show a reasonable feature of $I_{D}$ versus $V_{D}$ characteristics of a HEMT.

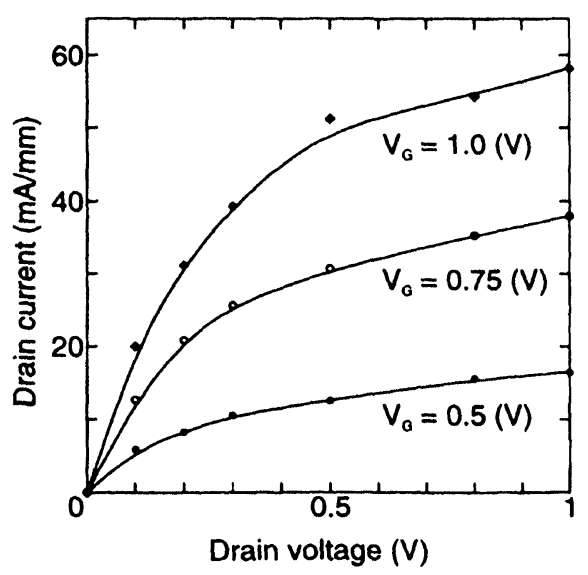

FIGURE $5 I_{D}$ versus $V_{D}$ characteristics of the HEMT obtained by the Monte Carlo simulation

\section{References}

[1] M. Tomizawa, A. Yoshii and K. Yokoyama, "Modeling for an $\mathrm{AlGaAs} / \mathrm{GaAs}$ heterostructure device using Monte Carlo simulation," IEEE Electron Device Lett., EDL-6, 332 (1985)

[2] U. Ravaioli and D. K. Ferry, "MODFET ensemble Monte Carlo model including the quasi-two-dimensional electron gas," IEEE Trans. Electron Devices, ED-33, 677 (1986)

[3] J.-R. Zhou and D. K. Ferry, "Modeling of quantum effects in ultrasmall HEMT device," IEEE Trans. Electron Devices. ED-40, 421 (1993)

[4] J. Lasserre and H. Tanimoto, "Hot electron drift velocity in AlGaAs heterojunctions," Jpn. J. Appl. Phys. 27, 924 (1988).

[5] P. J. Price, "Two-dimensional electron transport in semiconductor layers, I. Phonon scattering," Ann. Phys. 133, 217 (1981).

[6] F. Stern, "Self-consistent results for n-type Si inversion layers," Phys. Rev. B5, 4891 (1972). 

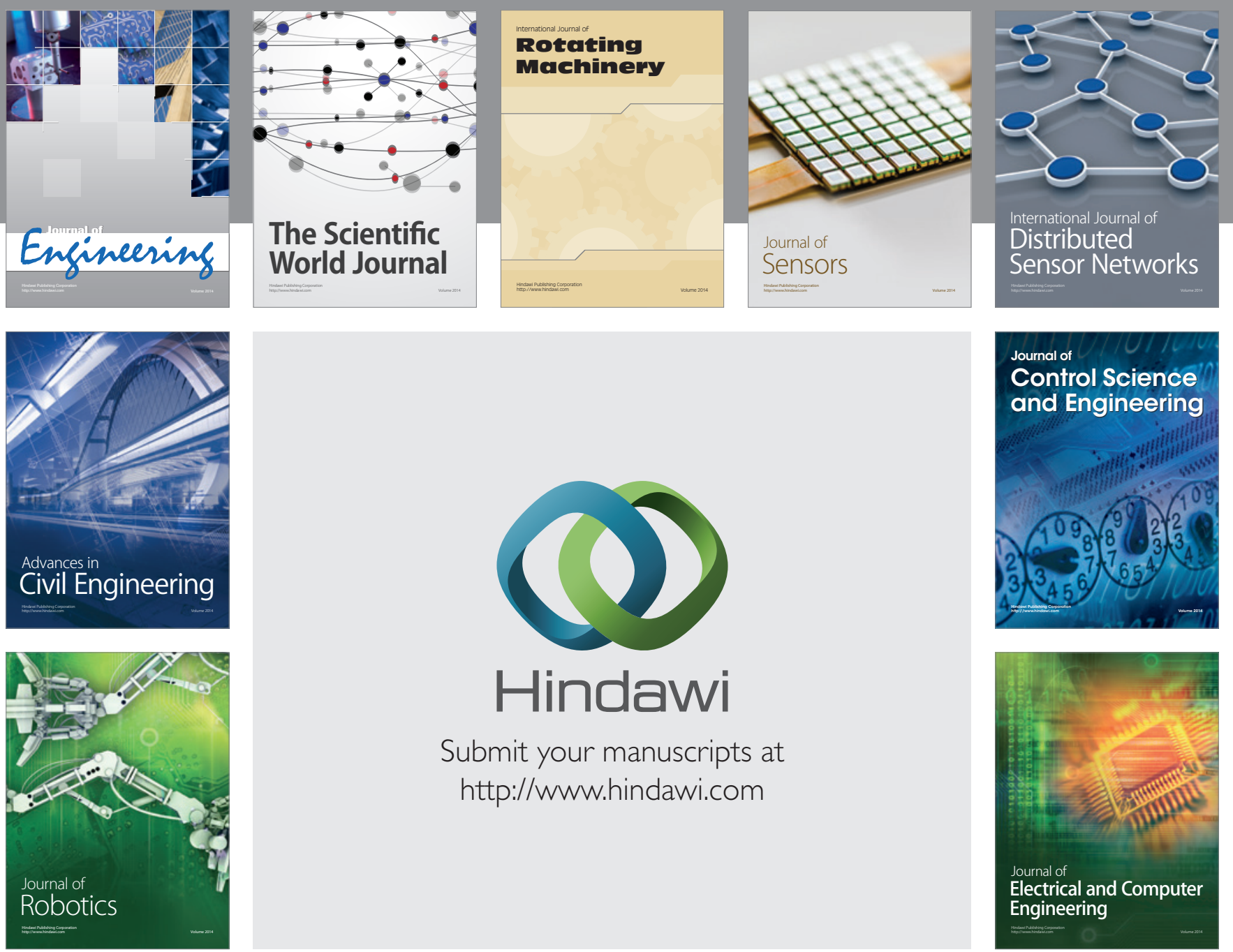

Submit your manuscripts at

http://www.hindawi.com
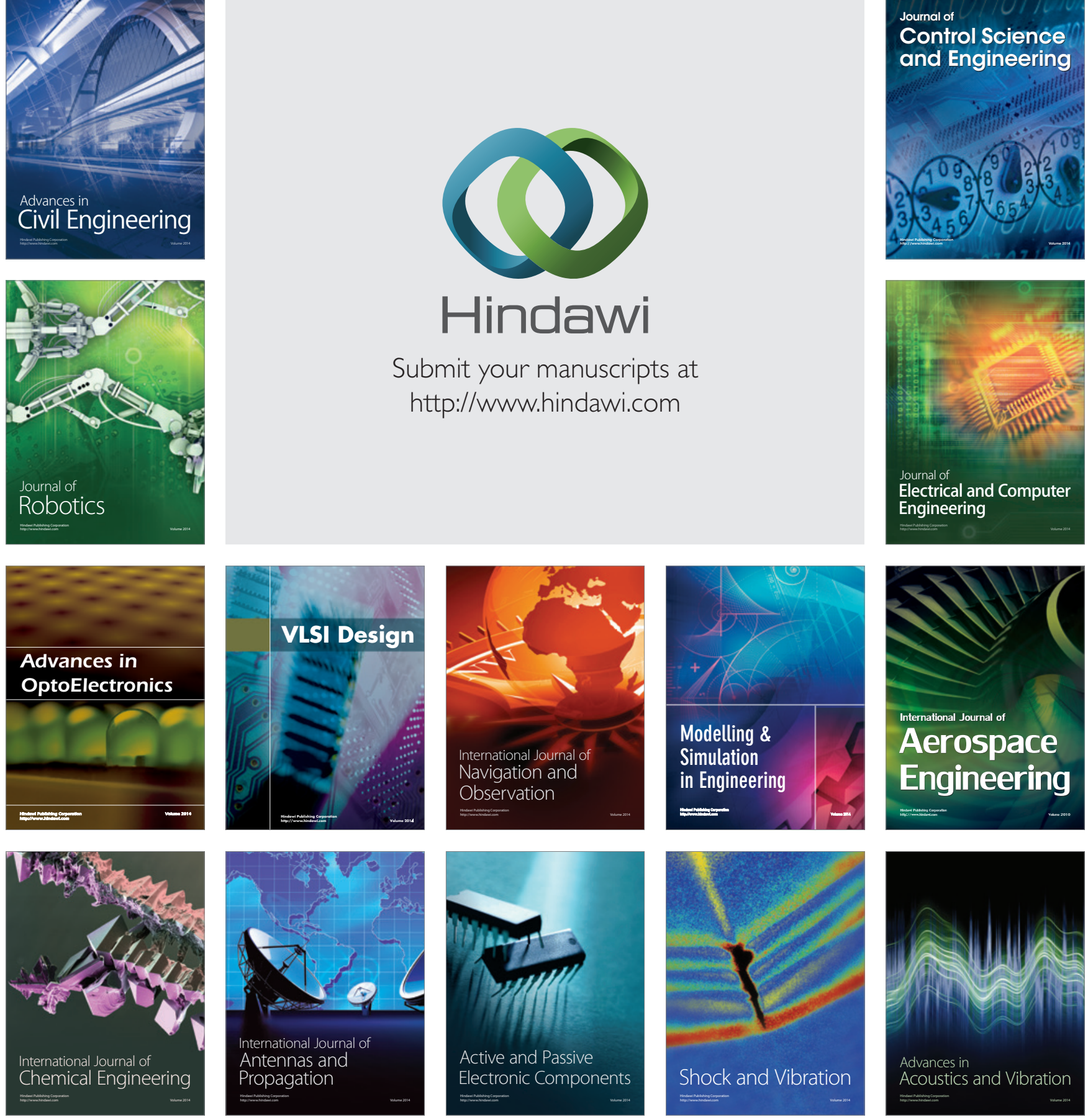